\title{
Comparison of serological responses in two different populations with pulmonary tuberculosis
}

\author{
Zaida Araujo/ ${ }^{+}$, Francesca Giampietro, Lucio Castellano Cançado' ${ }^{1}$, Mahavir Singh $^{2}$, Albina Wide $^{3}$
}

Laboratorio de Inmunología de Enfermedades Infecciosas, Instituto de Biomedicina ${ }^{3}$ Cátedra de Parasitología, Instituto de Medicina Tropical, Facultad de Medicina, Universidad Central de Venezuela, Caracas, Venezuela ${ }^{1}$ Universidade Federal do Triângulo Mineiro, Uberaba, MG, Brasil ' 2 IONEX Diagnostics and Therapeutics GMBH, Braunschweig, Germany

Observational studies on the humoural immune responses of the Warao indigenous people from Delta Amacuro, an isolated area, were compared with urban residents of the Venezuelan capital. Mycobacterium tuberculosisspecific reactivities (IgM, IgE, sIgA, IgG and IgG subclasses) were measured by ELISA using PPD and 38-kDa M. tuberculosis antigens. A total of 294 individuals were studied, 162 Warao (indigenous people) and 132 Creole (non-indigenous people). The patient group consisted of 87 Warao patients and 58 Creole patients, while the control group consisted of 75 Warao controls and 74 Creole controls. Combinations among the isotypes studied were performed. The findings showed that for the Warao people, sensitivity to the combination including anti-PPD IgG and IgE was $92.0 \%$, while for the Creole people, sensitivity to the combination including anti-PPD IgG but more so anti-PPD IgG1 and $\operatorname{IgG2}$ was $90.0 \%$. Simple tests were able to show higher specificities, which were populationspecific; specificities were anti-PPD IgG3, 100.0\% and anti-PPD IgM, 97.4\% for the Warao and Creole peoples, respectively. In conclusion, while simple tests reached high specificity, the multi-isotype tests improved sensitivity; the latter shows this approach may be useful in diagnostic testing.

Key words: tuberculosis - Warao - Creole - IgG subclasses - purified protein derivative - diagnosis

Tuberculosis (TB) diagnosis needs to be improved, as it largely depends upon clinical examination and radiographic findings, mainly confirmed by sputum smear microscopy and bacterial culture (Murray et al. 1980). The latter requires a long cultivation period and diagnostic confirmation still relies on sputum smear examinations. Many alternative methodologies have been applied in TB diagnosis, such as western blot, microscopic observed direct sensitivity culture, PCR and cell-mediated immune response reactions (Moore et al. 2006, Negi et al. 2006). These methods require trained personnel and specific laboratory conditions, which hinder their implementation in many areas of high TB endemicity (mainly low-income countries) and field work application. On the other hand, there are other options that are currently under evaluation, including antibody detection by serology. While specific antibody detection by serology is under evaluation for a definitive demonstration that the humoural response can be used as a tool for the diagnosis of TB, future studies should be carried out in order to combine serological and cellular methods, such as T.SPOT-TB or Quantiferon Gold assay, which

Financial support: FONACIT (S1-2000000667), BIOLAC/UNU

+Corresponding author: zaraujogarcia@yahoo.com

Received 10 April 2008

Accepted 24 October 2008 are considered to be approved tools. Regarding the immunodiagnosis of TB based on the $\mathrm{T}$ cell response to ESAT- 6 and purified protein derivative (PPD) antigens, recently it has been reported that the IFN- $\gamma$ assay using ESAT- 6 as an antigenic stimulus has the potential to be used as a tool for the immunodiagnosis of early TB in children (Van-Lume et al. 2008).

The demand for a rapid, reliable, cost-effective and easy TB diagnostic tool focuses on antibody detection by serology (Bothamley 1995). It has been reported that the isotypic restriction of antibodies correlates with the biochemical nature of antigens; most antibodies against proteins are of IgG1 and IgG3 isotypes, while in those against carbohydrates IgG2 is over-represented (Chiang et al. 1997, Gupta et al. 2005). This is reflected in vivo where, for instance, antibody responses to viral proteins are mainly of the IgG1 and IgG3 subclasses. In contrast, bacteria carbohydrates usually induce type 2 T-independent responses, mainly of the IgG1 and IgG2 isotypes. Several ELISA tests have been attempted and results have presented a large variability in their accuracy depending on the antigen employed (one alone or a pool) (Chiang et al. 1997, Gupta et al. 2005), the immunoglobulin (Ig) class, the subclass measured (Radin et al. 1983, Daniel \& Debane 1987, Pottumarthy et al. 2000, Conde et al. 2004, Imaz et al. 2004) and Mycobacterium tuberculosis strain variation (Alde et al. 1989, Zheng et al. 1994, Raja et al. 2002). This indicates either that other antigens need to be assessed for diagnosis or it reinforces the idea of differential antibody responses due to genetic ethnic variation (Demkow et al. 2004, Weldingh et al. 2005). In the latter, few studies have addressed the importance of the antibody response due to a different genetic background. 
Official data on the TB situation in Venezuela showed that between 1997-2001, the TB rate was between 93.281.0 per 100,000 inhabitants among the Warao people (MSAS 1999, Fernandez de Larrea et al. 2002, MSDS 2002). Both the Warao and Creole peoples have been tested for the human leukocyte antigen (HLA), HLA-A, B, HLA-Cw, HLA-DR and HLA-DR/DW 1-8 antigens. For the Warao, the findings confirmed the existence of the DR/DW dissociation previously observed in North American Indian, Japanese and Caucasian populations and showed inheritance and segregation of DW specificities (DW 8.3, DW 16, DW 22) defined only with homozygous typing cells of Warao origin. These data illustrated HLA haplotypes, linkage-disequilibrium and DR/DQ associations not seen previously in other human populations (Araujo et al. 2003). Data regarding the HLA class I and class II allele and haplotype distributions among Venezuelan Creoles showed that genes of Mongoloid, Negroid and Caucasoid origin have created a distinctive HLA genetic profile in this hybrid Creole population. The predominant HLA-B DQA1 DQB1 DRB1 haplotype is HLA-B44 DQA1*0201 DQB1*0201 DRB1*0701 (5.3\%). It is noteworthy that the HLA-A3 B7 DR2 and the HLA-A1 B8 DR3 linkage groups are part of conserved or ancestral haplotypes (Makhatadze et al. 1997).

In order to study the serological response in two populations with different genetic backgrounds, in the present work saliva $\operatorname{IgA}(\operatorname{sg} \mathrm{A})$ and serum $\operatorname{IgM}, \operatorname{IgE}$, $\operatorname{IgG}$ and $\mathrm{IgG} 1, \operatorname{IgG} 2$ and $\operatorname{IgG} 3$ subclass reactivities were measured against $M$. tuberculosis antigens in the Warao and Creole peoples.

\section{PATIENTS, MATERIALS AND METHODS}

Study population and clinical and epidemiological criteria - A prospective trial was performed, which included the Warao indigenous people, a population in a rural area isolated from contact with the tubercle bacillus for millennia until the mid-1960s. Within the Warao population, a high prevalence of active TB is observed, indicating a high susceptibility to disease, even among bacille Calmette-Guerin-vaccinated individuals. The humoural immune response of the Warao was compared with individual residents of the capital of Venezuela (urban area), the Creoles. A total of 294 adults aged from 15-60 years were studied: 162 Warao and 132 Creoles living in rural and urban areas, respectively. Individuals were grouped according to patient group: Warao patients $(\mathrm{WP}, \mathrm{n}=87)$, and Creole patients $(\mathrm{CP}, \mathrm{n}=58)$. These individuals had TB disease activity, where the clinical evaluation included recent weight loss or inadequate progress of weight gain, prolonged febrile syndrome, night sweats and coughing or wheezing for more than two weeks. The control group consisted of Warao healthy controls (WC, $n=75)$, and Creole healthy controls $(C C, n=74)$. These were individuals with lack of symptoms or without $\mathrm{TB}$ disease activity. The tuberculin skin test (TST) was performed on all the individuals of this study by a similar test previously described. The criterion for positive test reactivity was based on measurements of induration values $\geq 10 \mathrm{~mm}$ (Snider 1982, Arnadottir et al. 1996). For the confirmatory diagnosis of TB, smears from sputum were stained by the Ziehl-Neelsen direct method. For each sputum specimen, two tubes of modified Ogawa egg medium and Lowënstein-Jensen were inoculated, using the swab method of Kudoh and Kudoh (1974). Vaccination status was inferred from the presence of BCG scars; in Venezuela, the vaccination program has been conducted by the Tuberculosis National Program, which recommends the BCG immunization international policies, namely one dose of vaccine at the age of $0-5$ years old. Exclusion criteria included: (1) patients and healthy controls who are HIV positive; (2) patients taking immunosuppressive drugs (e.g., corticosteroids, azathioprine and cyclophosphamide) and (3) participants who did not sign an informed consent agreement.

Specific treatments were initiated in all newly identified cases of TB following the norms of the Tuberculosis National Program (MSAS 1999, MSDS 2002). This study was approved by the Ethical Committee of the Biomedicine Institute, Central University of Venezuela, (protocol number 2000000667/18/06/99) and written informed consent was voluntarily signed by all patients and control individuals.

Multiple Antigen Blot Assay (MABA) - MABA test was used to confirm mycobacteria-PPD and $38-\mathrm{kDa}$ as antigens for the purposes of studying the isotype reactivities (Noya \& Alarcón 1998). Briefly, MABA permits the simultaneous screening of different antigens, saliva or sera based on a dot-blot ELISA test. Using an acrylic device (Minibloter ${ }^{\circledR} 28$ S-L Immunetics Inc, Cambridge, MA, USA) containing at least 12 parallel troughs, antigens were distributed and immobilized onto a nitrocellulose membrane. Strips were cut perpendicularly and exposed to immune samples (sera and saliva diluted 1:400 and $1: 10$, respectively) for $1 \mathrm{~h}$ at $37^{\circ} \mathrm{C}$. Then these were washed four times and then incubated for $1 \mathrm{~h}$ at $37^{\circ} \mathrm{C}$ with peroxidase-conjugated monoclonal antibody antiIgG-gamma chain (Promega Corporation, USA) and peroxidase-conjugated monoclonal antibody anti-alpha chain IgA (Sigma-Aldrich, USA), which were used as the second antibodies. The reaction was developed by a chemiluminescent substrate, the results being recorded on film. Positive reactions to the different antigens are seen as small black squares on each strip.

Anti-PPD $\operatorname{Ig} G, \operatorname{Ig} M$ and $\operatorname{IgE}$ tests - ELISA was performed for the measurement of antibodies (IgG, IgM and $\mathrm{IgE}$ ) against PPD as previously reported (Araujo et al. 2004). Briefly, the levels of anti-PPD IgG, IgM and IgE were determined in serum by an ELISA as follows. Sera were isolated from venous blood obtained from controls and TB patients. Microtiter plates (Immunolon, Birmingham, UK) with 96 wells were coated with PPD (Statens Seruminstitut, Copenhagen), $(1 \mu \mathrm{g} / \mathrm{well}$ of each antigen in carbonate-bicarbonate buffer $\mathrm{pH}$ 9.6) overnight at $4^{\circ} \mathrm{C}$. Excess protein binding sites were blocked by incubation with $1 \%$ BSA in PBS at $37^{\circ} \mathrm{C}$ for $1 \mathrm{~h}$. Samples diluted 1:400 (for anti-PPD IgG) and 1:50 (for antiPPD IgM and IgE) in PBS containing 0.5\% BSA were added and plates were incubated for $1 \mathrm{~h}$ at $37^{\circ} \mathrm{C}$. The plates were washed four times, then incubated with the secondary antibodies: anti-human IgG HRP-conjugate 
(Promega Corporation, USA), anti-human IgM HRPconjugate (PIERCE Biotechnology) and anti-human IgE biotin-labelled (Vector Laboratories), incubated for $1 \mathrm{~h}$ at $37^{\circ} \mathrm{C}$ and washed four times. Substrate solution $(30 \mu \mathrm{L}$ of $30 \% \mathrm{H}_{2} \mathrm{O}_{2}$ and $10 \mathrm{mg}$ o-phenylenediamine dihydrochloride, Sigma-Aldrich, USA) in $25 \mathrm{~mL}$ citrate buffer, $\mathrm{pH} 5$ was added and incubated for 6 min at rt. Colour development was measured in an ELISA reader at $492 \mathrm{~nm}$.

Anti-38 kDa sIgA - The levels of SIgA in saliva were determined by a similar ELISA to that described for serum anti-PPD IgG. Microtiter plates were coated with $38 \mathrm{kDa}$ antigen (GBF and LIONEX GmbH, Braunschweig, Germany) overnight at $4^{\circ} \mathrm{C}(1 \mu \mathrm{g} /$ well of each antigen in carbonate-bicarbonate buffer $\mathrm{pH}$ 9.6). The saliva samples were diluted 1:10 and monoclonal antibody antihuman IgA peroxidase-conjugate (Sigma-Aldrich, USA) was used as the secondary antibody.

Anti-PPD IgG subclasses - The levels of anti-PPD IgG1, IgG2 and IgG3 subclasses were determined by a similar ELISA to that described for serum anti-PPD IgG. Briefly, microtiter plates (Immunolon, Birmingham, UK) with 96 wells were coated with PPD antigen $(1 \mu \mathrm{g} /$ well). The serum samples were diluted 1:50 and peroxidase-conjugate monoclonal antibodies anti-human IgG1, IgG2 and IgG3 (AP006, AP007 and AP008, The Binding Site, UK) were used as the secondary antibodies.

Other serological markers - HIV diagnostic testing was done with the Passive Particle Agglutination Test for the detection of antibodies to HIV-1 and/or HIV-2 from FUJIREBIO Diagnostics (Abbott ${ }^{\circledR}$ Laboratorie, Dainabot Co Ltd, Tokyo, Japan).

Statistical analysis - The statistical test used to compare the significance of the differences between the percentage values of individuals positive and negative for the TST was the Fisher's exact test. The reactivity was measured by enzyme linked immunosorbent assay and the differences in the isotype reactivity for each group were compared and evaluated using an unpaired Student's $t$ test; $\mathrm{p}$ values $<0.05$ were considered significant. R Development Core Team (2008) was used to highlight the outliers for showing the dispersion in the figures.

\section{RESULTS}

Bacteriological studies - The sensitivities of the smears were $70.0 \%$ and $64.0 \%$ for the Warao and Creole peoples, respectively, while culture test showed a sensitivity of $100.0 \%$ (Table).

Delayed type hypersensitivity and $B C G$ vaccination status - There was no statistically significant difference in relation to the TST reactivity between the WP $(80 / 87)$ and CP (47/58) groups. However, within control groups, the WC group (53/75) showed higher TST reactivity than the CC group $(34 / 74)(\mathrm{p}<0.001)$ (Table). The findings regarding the $\mathrm{BCG}$ vaccination status between the groups suggests that BCG vaccination does not have a significant effect on the incidence of the TST response (data not shown).

Reactivity response - The means of optical density \pm deviation density of the anti-PPD IgG, IgG subclasses, $\operatorname{IgM}, \operatorname{IgE}$ and anti-38kDa $\operatorname{sg} \mathrm{A}$ reactivities are shown in Fig. 1. The anti-PPD IgG showed higher reactivity in serum and anti-sIgA $38-\mathrm{kDa}$ in saliva when MABA was used to select the antigen for studying the isotype reactivity (data not shown). The anti-PPD IgG reactivity evaluation showed that WP presented higher reactivity $(1.010 \pm 0.348)$ than respective controls, WC $(0.592$ $\pm 0.139)$ and $C C(0.669 \pm 0.222)(\mathrm{p}<0.0001)$ (Fig. 1A). Also for anti-PPD IgM reactivity, it was observed that WP presented a significantly higher reactivity $(0.365 \pm$

TABLE

Comparative study of sensitivity and specificity of the different methods for tuberculosis diagnosis between Warao and Creole populations

\begin{tabular}{|c|c|c|c|c|}
\hline \multirow[b]{3}{*}{ Specific / Reactivity } & \multicolumn{2}{|c|}{ Warao } & \multicolumn{2}{|c|}{ Creole } \\
\hline & Sensitivity & Specificity & Sensitivity & Specificity \\
\hline & \multicolumn{2}{|c|}{$\%$} & \multicolumn{2}{|c|}{$\%$} \\
\hline Bacteria + & $70.0^{a}$ & 67.0 & $64.0^{d}$ & 84.0 \\
\hline Bacteria /Culture + & 100.0 & 100.0 & 100.0 & 100.0 \\
\hline $\operatorname{TST}^{g}(+)$ & 92.0 & $29.0^{c}$ & 81.0 & $55.0^{f}$ \\
\hline Anti-PPD IgG & 77.0 & 65.0 & 76.0 & 50.0 \\
\hline Anti-PPD IgM & 9.6 & 96.4 & 17.3 & 97.4 \\
\hline Anti-PPD IgE & 62.0 & 64.0 & 16.0 & 80.0 \\
\hline Anti-PPD IgG1 & 45.0 & 89.0 & 38.0 & 86.0 \\
\hline Anti-PPD IgG2 & 44.0 & 67.0 & 55.0 & 88.0 \\
\hline Anti-PPD IgG3 & 21.0 & 100.0 & 33.0 & 89.0 \\
\hline Anti-38 kDa sIgA & 16.0 & 90.0 & 2.0 & 93.0 \\
\hline Anti-PPD IgG and IgE & $92.0^{b}$ & 37.4 & & \\
\hline Anti-PPD IgG, IgG1 and IgG2 & $90.0^{e}$ & 43.2 & & \\
\hline
\end{tabular}

$a, b: \mathrm{p}<0.0004 ; \mathrm{c}, d, e, f: \mathrm{p}<0.001 ; \mathrm{g}$ : the tuberculin skin tests were performed using two tuberculin units of purified protein derivative (PPD) of Mycobacterium tuberculosis; strain RT-23 from the Statens Serum Institute in Copenhagen. 
A

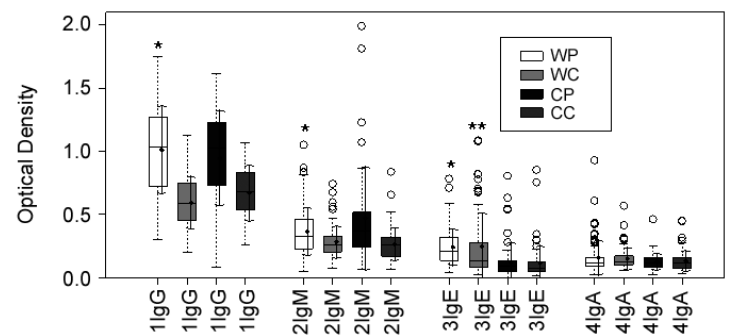

B

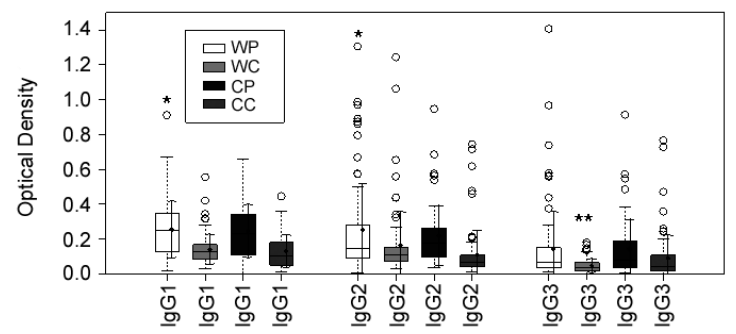

Fig. 1: A: reactivity detections of anti-purified protein derivative (PPD) IgG, IgM, IgE and anti-38kDa sIgA. The results are shown as absolute value means $\pm \mathrm{SD}$ of the isotype reactivity in each group. Warao patients (WP): $\mathrm{n}=87$; Warao controls (WC): $\mathrm{n}=75$; Creole patients (CP): $\mathrm{n}=58$; and Creole controls (CC): $\mathrm{n}=74 ; *$ and $* *$ : the significance between groups. For anti-PPD IgG: * WP \& WC and CC; for anti-PPD IgM: * WP \& WC and CC; for anti-PPD IgE: * WP \& $\mathrm{CP}$ and $\mathrm{CC}$, ** WC \& $\mathrm{PC}$ and $\mathrm{CC}$; $\mathrm{B}$ : reactivity detections of anti-PPD IgG1, IgG2 and IgG3; * and **: the significance between groups. For anti-PPD IgG1: * WP \& WC and CC; for anti-PPD IgG2: * WP \& WC and CC; for anti-PPD IgG3: ** WC \& WP and CP and CC; O: the dispersion in the figures.

$0.193)$ than respective controls, WC $(0.281 \pm 0.074)$ and $\mathrm{CC}(0.264 \pm 0.133)(\mathrm{p}<0.0005$ and $\mathrm{p}<0.0002$, respectively) (Fig. 1A). Concerning the IgE antibody response, serum anti-PPD IgE reactivity analysis showed that the Warao people reacted more than the Creole people, independently of whether they were patients or controls (Fig. $1 \mathrm{~A})$, WP $(0.241 \pm 0.146)$ and $\mathrm{WC}(0.247 \pm 0.033)$ than CP $(0.127 \pm 0.035)$ and $\mathrm{CC}(0.115 \pm 0.136)(\mathrm{p}<0.0001)$ (Fig. 1A). Regarding anti-38kDa sIgA reactivity, a statistically significant difference was not observed between the groups (Fig. 1A).

Serum anti-PPD IgG1 analysis showed that WP presented a significantly higher IgG1 reactivity $(0.255 \pm$ $0.167)$ than WC $(0.140 \pm 0.156)$ and $C C(0.130 \pm 0.096)$ $(\mathrm{p}<0.0001)$ (Fig. 1B). For anti-PPD IgG2 reactivity, it was observed that WP presented a significantly higher $\operatorname{IgG} 2$ reactivity $(0.255 \pm 0.267)$ than respective controls, WC $(0.165 \pm 0.159)$ and CC $(0.111 \pm 0.142)(\mathrm{p}<0.001$ and $\mathrm{p}<0.0001$, respectively) (Fig. 1B). There was no anti-PPD IgG3 reactivity in WC $(0 \pm 0)$ as compared to WP $(0.142$ $\pm 0.0 .219), \mathrm{CP}(0.145 \pm 0.108)$ and $\mathrm{CC}(0.090 \pm 0.137)(\mathrm{p}<$ $0.0002, \mathrm{p}<0.0001$ and $\mathrm{p}<0.005$, respectively) (Fig. 1B).

The antibody reactivity to $M$. tuberculosis antigens after and before treatment is shown in Fig. 2. We observed a statistically significant difference between antiPPD IgG reactivity after treatment $(0.576 \pm 0.205)$ and before treatment $(0.902 \pm 0.072)(\mathrm{p}<0.008)$ (Fig. 2A). For anti-PPD IgE, the higher reactivity found after treat-
A

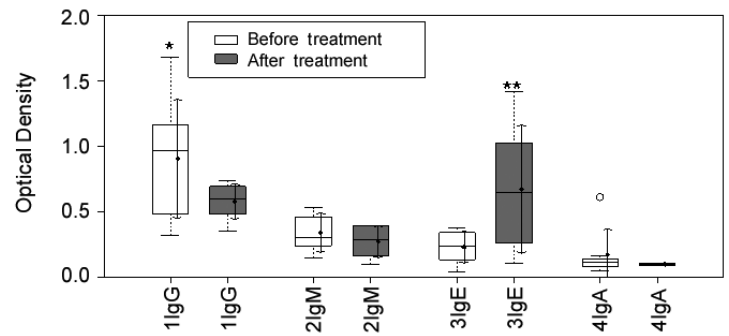

B

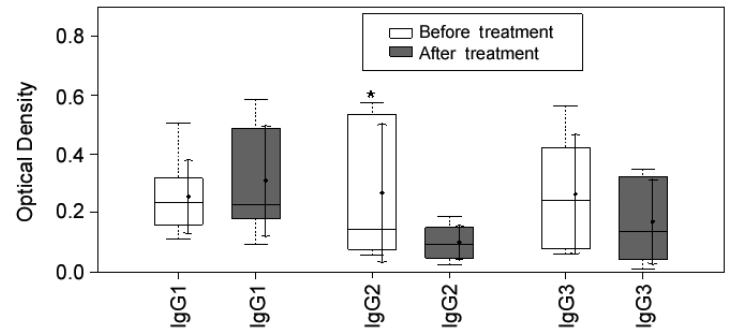

Fig. 2: A: reactivity detections of anti-purified protein derivative (PPD) IgG, IgM, IgE and anti-38kDa sIgA before and after treatment in Warao patients. Data representing the value means $\pm \mathrm{SD}$ of the isotype reactivity; * and **: the significance of anti-PPD IgG and antiPPD IgE reactivities, respectively between before and after treatment; $B$ : reactivity detections of anti-PPD IgG subclasses; * significance of anti-PPD IgG2 between before and after treatment; $\bigcirc$ : the dispersion in the figures.

ment $(0.669 \pm 0.465)$ was statistically significant compared to before treatment $(0.225 \pm 0.184)(\mathrm{p}<0.02)$ (Fig. $2 \mathrm{~A})$. There was not a statistically significant difference concerning anti-PPD IgM, anti-PPD IgG1, anti-PPD IgG3 and anti-38-kDa $\operatorname{sgA}$ reactivities after treatment as compared to before treatment (Fig. 2A), while we did observe a statistically significant difference between anti-PPD $\operatorname{IgG} 2$ reactivity after $(0.100 \pm 0.026)$ and before treatment $(0.267 \pm 0.008)(\mathrm{p}<0.0001)$ (Fig. $2 \mathrm{~B})$.

Sensitivity and specificity tests - The sensitivity and specificity of the different methods are shown in the Table. We observed high specificity between the methods, two methods were especially highly specific, anti-PPD $\operatorname{IgG3}$ and anti-PPD IgM $(100.0 \%$ in the Warao people and $97.4 \%$, in the Creole people, respectively) (Table). However, we found low sensitivity between the methods, anti$38 \mathrm{kDa} \operatorname{sg} \mathrm{A}(2.0 \%$ in the Creole people) and anti-PPD $\operatorname{IgG}(77.0 \%$ in the Warao people). Among the combinations performed among all isotypes, in the Warao people the anti-PPD IgG and anti-PPD IgE combination and in the Creole people, the anti-PPD IgG, anti-PPD IgG1 and anti-PPD IgG2 combination detected the largest number of patients which brought an improved sensitivity level of $92.0 \%$ and $90.0 \%$, respectively, even though specificity levels dropped to $37.4 \%$ and $43.2 \%$, respectively (Table). In both patient groups, there were statistically significant differences between the sensitivities of the combination methods and the smear methods, $92.0 \%$ versus $70.0 \%, \mathrm{p}<$ 0.0004 , and $90.0 \%$ versus $64.0 \%, \mathrm{p}<0.001$, in the Warao and Creole groups, respectively (Table). 


\section{DISCUSSION}

The present work tried to reinforce the use of multiantigen and multi-isotype assays as the central rationale to develop a test for TB to be used effectively in different settings in Venezuela. The findings showed that combinations among the isotypes studied improved the sensitivity, even though the specificity dropped to lower levels; for the Warao people, sensitivity of the combination including anti-PPD IgG and IgE was at $92.0 \%$, while for the Creole people, sensitivity of the combination that included anti-PPD IgG but more so anti-PPD IgG1 and $\mathrm{IgG} 2$ was at $90.0 \%$. In contrast, simple tests were able to show higher specificities, which were populationspecific, anti-PPD IgG3 (100.0\%) and anti-PPD IgM $(97.4 \%)$ for the Warao and Creole peoples, respectively. Regarding the $\mathrm{IgG}$ specific response, the results showed that the anti-PPD IgG responses were serologically indistinguishable between Warao and Creole untreated TB patients. These results are in concordance with studies showing that patients with active TB clearly had higher levels of IgG antibody to PPD antigen than did a healthy control group (Radin et al. 1983, Hussain et al. 1995).

It has been proposed by the WHO that new diagnostic methods should have sensitivity at least higher than bacilloscopy (WHO 2003). The sensitivities of the microscopic examination of sputum for acid-fast bacilli were statistically low in the WP and CP groups as compared to the combination methods. In a previous study conducted with Warao children, we found for anti-PPD IgG a low sensitivity of $38.2 \%$ and a high specificity of 95.8\% (Araujo et al. 2004). One report describes the high and low sensitivity of anti-PPD IgG test (Lyashchenko et al. 1998); these different findings can be explained since it has been reported that serum IgG antibodies are produced against a variety of epitopes on the antigen surface. Also, the number and the species of serologically reactive antigens varied greatly from individual to individual, and the level of specific antibodies could also vary with the genetic background or the age of individuals (Lyashchenko et al. 1998).

The anti-PPD IgM methods showed low sensitivities, while specificities were higher for both populations. The latter correlates with the results reported on the commercial methods, which use recombinant mycobacterial antigens. These commercial methods available for TB serological diagnosis based on IgM reactivity show lower sensitivity rates for both adults and children than tests based on other antibody classes, although specificity has remained at elevated rates (Pottumarthy et al. 2000, Imaz et al. 2004).

The anti-38kDa sIgA method was included in our study due to the fact that it provided an improvement in the diagnosis of TB in Warao children (Araujo et al. 2004). A combination including anti-PPD $\operatorname{IgG}, \operatorname{IgE}$ and anti-38kDa $\operatorname{sg} \mathrm{A}$ detected a larger number of child patients and allowed us to obtain a sensitivity level of $64.7 \%$ and a specificity of $81.8 \%$ (Araujo et al. 2004). In the present study a major percentage of WP elicited saliva positive reactivity, anti-38kDa $\operatorname{sIgA}$, as compared to the Creole people; these methods were not very sensi- tive, but highly specific. These findings correlate with previous results obtained in Warao children, where the anti-38kDa sIgA method showed high specificity and low sensitivity (Araujo et al. 2004). Few studies have addressed anti-38kDa sIgA reactivity, although it has been reported to have sensitivity around $80 \%$ using the $38-\mathrm{kDa}$ antigen to test sera samples instead of saliva (Young et al. 1986, Uma et al. 2001). Regarding the anti-PPD IgG subclass reactivities, higher percentages of both WP and $\mathrm{CP}$ showed anti-PPD IgG1 and $\mathrm{IgG} 2$ reactivities than control groups; the latter was highly reactive in the Creole people as compared to the Warao people. It has been reported that antibody responses to bacterial carbohydrates are mainly of the IgG1 and IgG2 subclasses (Gupta et al. 2005). The importance of IgG2 in human TB is not evident; the authors reported that a few cases of active TB showed moderately increased IgG2, while in relapse and chronic TB cases with reactivity to $\operatorname{IgG} 2$ these levels were decreased. Therefore, IgG2 subclass could not differentiate between TB cases and healthy controls with latent infection (Gupta et al. 2005). The latter was not in concordance with our results for the Warao people, as WP presented a significant higher reactivity than the respective control groups studied.

The present results regarding anti-PPD IgG3 reactivity are in concordance with a report that shows that in TB patients, both anti-PPD IgG1 and IgG3 tests reacted more often than in controls (Hussain et al. 1995, Gupta et al. 2005). Within the Warao people, the anti-PPD IgG3 method showed the highest specificity at $100 \%$, even though sensitivity levels dropped to $21 \%$. These findings suggest that there is no basis for a useful diagnostic test, but it could be of use in confirming that no TB cases among the people from this population resulted from false positive cases.

When IgG, IgM, sIgA and IgG subclass reactivities were evaluated before and after treatment in the Warao population, it was found that two of these, $\operatorname{IgG}$ and $\operatorname{sIg} \mathrm{A}$, were affected by anti-TB treatment. The results permit us to suggest that these isotypes are markers of active infection and could be predictive of treatment outcome in this population, such as has been reported by others authors, who have suggested that the levels of antibodies to M. tuberculosis represent serological correlates of active disease since these were affected in an antigen-specific fashion by anti-TB treatment (Azzurri et al. 2006).

Since it is known that poor sensitivity tests may be due to substantial and unexplained heterogeneity of the humoural response to M. tuberculosis (Hussain et al. 1995), it seems probable that the development of either multi-antigen tests or multi-isotype tests could optimize both sensitivity and specificity. The present findings showed that the combination methods were able to optimize sensitivity; for the Warao people, sensitivity of the combination including anti-PPD $\operatorname{IgG}$ and $\operatorname{IgE}$ was at $92.0 \%$, while for the Creole people, sensitivity of the combination including anti-PPD IgG but more so antiPPD IgG1 and IgG2 was at $90.0 \%$. In contrast, simple tests were able to show higher specificities, which were population-specific, with anti-PPD IgG3 at $100.0 \%$ and anti-PPD IgM at $97.4 \%$ for the Warao and Creole peo- 
ple, respectively. In a report on the antibody responses to CFP-21, ESAT-6, MPT-63 and MPT-64 antigens, it was found that sensitivity to the multi-antigen complex was higher than for the four single antigens and that the specificity was at $100 \%$ (Wang et al. 2005).

The combination methods utilized in the present study should be extended to other TB endemic regions of Venezuela for the evaluation of both the sensitivity and specificity of the methods. Taking into account that the combination methods included PPD, a crude antigen that is present in other Mycobacterium species, such as non TB mycobacteria and Mycobacterium leprae, further studies testing in parallel patients with leprosy and using different antigens such as rESAT-6 or rAg85 are necessary. In addition, these methods must be combined with any of the sensitive and approved tools, such as IFN- $\gamma$ release assay, T.SPOT-TB or Quantiferon Gold assay; if these tests are in agreement with the methods studied here, it could reinforce the use of the multi-antigen and multi-isotype tests performed in this study. Finally, while the multi-isotype methods improved sensitivity, simple methods allowed for maximum specificity to be obtained. Since our results show that both a combination method and a simple method yield different responses in specific populations, the variability of the humoural immune response to $M$. tuberculosis must be taken into account in order to optimize sensitivity, especially in populations with different genetic backgrounds.

\section{ACKNOWLEDGEMENTS}

To Komal Roy Siripaul, for critical review of the manuscript.

\section{REFERENCES}

Alde SLM, Piñasco HM, Pelosi FR, Budani HF, Palma-Beltran OH, Gonzalez Montaner LJ 1989. Evaluation of an enzyme-linked immunosorbent assay (ELISA) using an IgG antibody to Mycobacterium tuberculosis antigen 5 in the diagnosis of active tuberculosis in children. Am Rev Respir Dis 139: 748-751.

Araujo Z, de Waard JH, Fernández de Larrea C, López D, Fandiño C, Maldonado A, Hernández E, Ocaña Y, Ortega R, Singh M, Ottenhoff THM, Arend SM, Convit J 2004. Study of the antibody response against Mycobacterium tuberculosis antigens in Warao Amerindian children in Venezuela. Mem Inst Oswaldo Cruz 99: 517-524.

Araujo Z, Fernández de Larrea C, López D, Fandiño C, Chirinos M, Convit J, Debora I, de Waard JH 2003. Hematological values among Warao Indians with tuberculosis from the Orinoco delta of Venezuela. Acta Cient Venez 54: 247-253.

Arnadottir T, Rieder HI, Trébuq A, Waaler H 1996. Guidelines for conducting tuberculin skin test surveys in high prevalence countries. Int J Tuberc Lung Dis 77: 1-20.

Azzurri A, Kanaujia GV, Sow OY, Bah B, Diallo A, Del Prete G, Gennaro ML 2006. Serological markets of pulmonary tuberculosis and of response to anti-tuberculosis treatment in a patient population in Guinea. Int J Immunopathol Pharmacol 19: 199-208.

Bothamley GH 1995. Serological diagnosis of tuberculosis. Eur Respir J 8 (Suppl. 20): 676s-688s.

Chiang IH, Sou J, Bai KJ, Lin TP, Luh KT, Yu CJ, Yang PC 1997. Serodiagnosis of tuberculosis. A study comparing three specific mycobacterial antigens. Am J Respir Crit Care Med 156: 906-911.

Conde MB, Suffys P, Lapa e Silva JR, Kritski AL, Dorman SE 2004. Immunoglobulin A (IgA) and $\operatorname{IgG}$ immune responses against p-90 antigen for diagnosis of pulmonary tuberculosis and screening for Mycobacterium tuberculosis infection. Clin Diagn Lab Immunol 11: 94-97.

Daniel TM, Debane SM 1987. The serodiagnosis for tuberculosis and other mycobacterial diseases by enzyme-linked immunosorbent assay. Am Rev Respir Dis 135: 1137-1151.

Demkow U, Ziólkowski J, Filewska M, Bialas-Chromiec B, Zielonka T, Michalowska-Mitczuk D, Kuś J, Augustynowicz E, Zwolska Z, Skopińska-Rózewska E, Rowińska-Zakrzewska E 2004. Diagnostic value of different serological tests for tuberculosis in Poland. J Physiol Pharmacol 55: 57-66.

Fernández de Larrea C, Fandiño C, López D, del Nogal B, Rodríguez N, Convit J, Araujo Z, de Waard JH 2002. Childhood tuberculosis in the Warao population in Venezuela. Invest Clin 43: 35-48.

Gupta S, Shende N, Bhatia AS, Kumar S, Harinath BC 2005. IgG subclass antibody response to mycobacterial serine protease at different stages of pulmonary tuberculosis. Med Sci Monit 11: 585-588.

Hussain R, Dawood G, Abrar N, Toosi Z, Minai A, Dojki M, Ellner J 1995. Selective increase in antibody isotypes and immunoglobulin $\mathrm{G}$ subclass response to secreted antigen in tuberculosis patients and healthy household contacts of the patients. Clin Diagn Lab Immunol 2: 726-732.

Imaz MS, Comini MA, Zerbini E, Sequeira MD, Latini O, Claus JD, Singh M 2004. Evaluation of commercial enzyme-linked immunosorbent assay kits for detection of tuberculosis in argentinean population. J Clin Microbiol 42: 884-887.

Kudoh S, Kudoh T 1974. A simple technique for culturing tubercle bacilli. Bull World Health Organ 51: 71-82.

Lyashchenko K, Colangeli R, Houde M, Al Jahdali H, Menzies D, Gennaro ML 1998. Heterogeneous antibody responses in tuberculosis. Infect Immun 66: 3936-3940.

Makhatadze NJ, Franco MT, Layrisse Z 1997. HLA class I and class II allele and haplotype distribution in the venezuelan population. Hum Immunol 55: 53-58.

Moore DAJ, Evans CAW, Gilman RH, Caviedes L, Coronel J, Vivar A, Sanchez E, Piñedo Y, Saravia JC, Salazar C, Oberhelman R, Hollm-Delgado MG, LaChira D, Escombe AR, Friedland JS 2006. Microscopic-observation drug-susceptibility assay for the diagnosis of TB. N Engl J Med 355: 1539-1550.

MSAS - Ministerio de Sanidad y Asistencia Social 1999. Programa Integrado de Control de la Tuberculosis. Seminario Técnico-Administrativo, Caracas, $150 \mathrm{pp}$.

MSDS - Ministerio de Salud y Desarrollo Social 2002. Evaluación del Programa Nacional de Control de la Tuberculosis. Año Evaluado 2001, Caracas, $201 \mathrm{pp}$.

Murray PR, Elmore C, Krogstad DJ 1980. The acid-fast stain: a specific and predictive test for mycobacterial disease. Ann Intern Med 92: 512-513.

Negi SS, Anand R, Basir SF, Pasha ST, Gupta S, Khare S, Lal S 2006. Protein antigen $\mathrm{b}(\mathrm{Pab})$ based PCR test in diagnosis of pulmonary and extra-pulmonary tuberculosis. Indian J Med Res 124: 81-88.

Noya O, Alarcón de Noya B 1998. The multiple antigen blot assay MABA a simple immunoenzymatic technique for simultaneous screening of multiple antigens. Immunol Lett 63: 173-176.

Pottumarthy S, Wells VC, Morris AJ 2000. A comparison of seven tests for serological diagnosis of tuberculosis. J Clin Microbiol 38: $2227-2231$

Radin RC, Zeiss CR, Phair JP 1983. Antibodies to purified protein derivative in different immunoglobulin classes in the diagnosis of tuberculosis in man. Int Arch Allergy Immunol 70: 25-29. 
Raja A, Uma Devi KR, Ramalingam B, Brennan PJ 2002. Immunoglobulin $\mathrm{G}, \mathrm{A}$ and $\mathrm{M}$ responses in serum and circulating immune complex elicited by the 16-kilodalton antigen of tuberculosis. Clin Diagn Lab Immunol 9: 308-312.

Snider DE 1982. The tuberculin skin test. Am Rev Respir Dis 125: 102-104.

Uma Devi KR, Ramalingam B, Brennan PJ, Narayanan PR, Raja A 2001. Specific and early detection of IgG, IgA and IgM antibodies to tuberculosis $38-\mathrm{kDa}$ antigen in pulmonary tuberculosis. Tuberculosis 81: 249-253.

Van-Lume DSM, de Souza JR, Melo WG, Melo VL, Cabral MML, Rego JC, Schindler HC, Abath FGC, Montenegro SML 2008. Preliminary results in the immunodiagnosis of tuberculosis in children based on T cell responses to ESAT- 6 and PPD antigens. Mem Inst Oswaldo Cruz 103: 401-404.

Wang BL, Xu Y, Li ZM, Xu YM, Weng XH, Wang HH 2005. An- tibody response to four secretory proteins from Mycobacterium tuberculosis and their complex antigen in TB patients. Int $J$ Tuberc Lung Dis 9: 1327-1334.

Weldingh K, Rosenkrands I, Okkels LM, Doherty TM, Andersen P 2005. Assessing the serodiagnostic potential of 35 Mycobacterium tuberculosis proteins and identification of four novel serological antigens. J Clin Microbiol 43: 57-65.

WHO - World Health Organization 2003. Global tuberculosis control. WHO, Geneve, p. 1-49.

Young D, Kent L, Rees A, Lamb J, Ivanyi J 1986. Immunological activity of a 38-kilodalton purified from Mycobacterium tuberculosis. Infect Immun 54: 177-183.

Zheng YJ, Wang RH, Lin YZ, Daniel TM 1994. Clinical evaluation of the diagnostic value of measuring IgG antibody to three mycobacterial antigen preparations in the capillary blood of children with tuberculosis and control subjects. Int J Tuberc Lung Dis 75: 366-370. 